\title{
Ventaja competitiva de Intel en el segmento de los microprocesadores
}

\author{
Intel's competitive advantage in the segment of the microprocessors
}

Recibido: 02-07-2019 • Aprobado: 29-07-2020 • Página inicial: 135 - Página final: 151

\author{
Laura Melina Rodríguez Gutiérrez* \\ José G. Vargas Hernández**
}

\begin{abstract}
Resumen: el presente trabajo tiene el objetivo de analizar cómo la empresa Intel Corporation se ha desarrollado a nivel mundial y cuál es su ventaja competitiva con respecto a los competidores en el mercado. El análisis se hará desde un punto de vista basado en la industria, identificando las cinco fuerzas de Michael Porter, lo cual brinda una evaluación de las fortalezas de los factores externos de la compañía para poder así definir estrategias ante las amenazas. Así mismo, se buscará cuál es la ventaja competitiva de Intel, dado su dominio actual en el segmento de las computadoras personales ante los cambios tecnológicos y los nuevos segmentos de la industria de los semiconductores. La hipótesis: Intel cuenta con ventaja competitiva, a través del volumen de producción, de las alianzas con fabricantes de PC y de las fábricas propias (Fabs) para producción de procesadores, que le permite entrar con mayor facilidad a los nuevos segmentos de tecnología. Y como objetivo: Analizar la ventaja competitiva de Intel a través de las cinco fuerzas de Porter.
\end{abstract}

Palabras clave: Intel; mercado; estrategia; cinco fuerzas; oligopolio; industria.

\begin{abstract}
The present work has the objective of analyzing how the company Intel Corporation has developed worldwide and what is its competitive advantage with respect to competitors in the market. The analysis will be made from an industry-based point of view, identifying the five forces of Michael Porter, which provides an assessment of the strengths of external factors to the company in order to define strategies against threats. What is Intel's competitive advantage given its current dominance in the personal computer segment in the face of technological changes and new segments of the semiconductor Industry? The hypothesis: Intel has a competitive advantage over production volume, alliances with PC manufacturers, own factories (Fabs)for the production of processors, which allows it to enter with greater ease the new technology segments, and as a goal: to analyze Intel's competitive advantage through Porter's five forces.
\end{abstract}

Keywords: Intel; market; strategy; five forces; oligopoly; industry.

JEL: L11 - L13 - L22

\footnotetext{
* Magister en Negocios y Estudios Económicos. Centro Universitario de Ciencias Económico-Administrativas. Universidad de Guadalajara, México. Lauritamrg@gmail.com. 


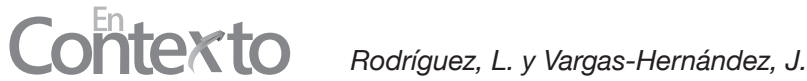

\section{Vantagem competitiva da Intel no segmento dos microprocessadores}

\begin{abstract}
Resumo: o presente trabalho tem o objetivo de analisar como a empresa Intel Corporation tem se desenvolvido mundialmente e qual sua vantagem competitiva em relação aos concorrentes no mercado. A análise será feita do ponto de vista da indústria, identificando as cinco forças de Michael Porter, que fornece uma avaliação dos pontos fortes dos fatores externos da empresa para definir estratégias contra ameaças. Da mesma forma, a vantagem competitiva da Intel será buscada, dado seu domínio atual no segmento de computadores pessoais em face das mudanças tecnológicas e novos segmentos da indústria de semicondutores. A hipótese: a Intel tem uma vantagem competitiva, por meio do volume de produção, alianças com fabricantes de PCs e fábricas próprias (Fabs) para a produção de processadores, o que lhe permite entrar com mais facilidade em novos segmentos de tecnologia. E como objetivo: Analisar a vantagem competitiva da Intel por meio das cinco forças de Porter.
\end{abstract}

Palavras-chave: Intel; mercado; estratégia; cinco forças; oligopólio; indústria. 


\section{Introducción}

El presente documento está destinado al análisis de la empresa Intel y cómo esta ha venido desarrollándose como una empresa en el mercado de semiconductores, los procesadores Intel pueden encontrarse en las computadoras personales. Por su alta producción, calidad, innovación que ha venido manejando durante varios años desde su fundación se ha colocado como una empresa líder en su rama, además de consolidarse como una empresa reconocida a nivel mundial. Junto con Intel existe AMD (Advanced Micro Devices), siendo esta la principal competencia para Intel en la rama de computadoras personales. Semmler (2010) coloca a Intel como una empresa que por su estructura de mercado se cataloga como un oligopolio, lo cual se explicará con detalle, profundizando en los puntos que señala este autor.

Además, se analizará cómo Intel Corporation se ha desempeñado para poder obtener una ventaja competitiva frente a sus rivales; como referencia tomaremos a Porter $(1981 ; 2008)$; quien nos hace su contribución en esta metodología con su investigación, la cual estudia las técnicas de análisis de la industria, llamada las cinco fuerzas o los diamantes de Porter. Estas se identifican como: rivalidad entre competidores, amenaza de una entrada potencial, el poder de negociación de los proveedores, el poder de negociación de los compradores, amenaza de los productos sustitutos; dichas fuerzas se analizarán más adelante con el objetivo de comprender en qué consiste cada una de ellas y poder identificar las características que posee cada una y cómo estas están relacionadas e implementadas por la compañía Intel Corporation.

Esta investigación concluye con un análisis de las ventajas que Intel tiene en la actualidad y cómo se está preparando para ingresar a nuevos mercados que están en auge, como la cartera tecnológica centrada en los datos para la inteligencia artificial, el análisis y la nube.

\section{Metodología}

Dada la naturaleza de esta investigación, el método que se empleará será el analítico, el cual contempla habilidades de pensamiento crítico, evaluación de los hechos que se están llevando a cabo y comprenderlos. Así como analizar los datos recopilados, que son herramientas importantes para examinar nuestra hipótesis, y poder ser capaz de llegar a una conclusión sobre el tema tratado. 


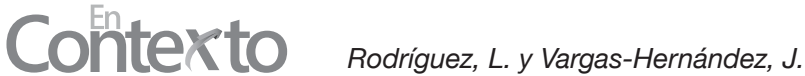

\section{Resultados}

\section{Antecedentes}

Intel Corporation es un fabricante líder de semiconductores en el mundo, esta compañía estadounidense es la creadora de la serie de procesadores $\mathrm{x} 86$, dichos procesadores son comúnmente encontrados en la mayoría de las computadoras personales (Intel, 2019). La compañía fue establecida en 1968 por Robert N. Noyce, cofundador del circuito integrado y Gordon E. Moore, un colega de la compañía Noyce's de Fairchild Semiconductor. Dado que el nombre de Moore Noyce ya estaba registrado como marca, optaron por llamar a la compañía Intel, nombre acrónimo de "Integrated Electronics" (Intel, 2019).

Misión: utilizar el poder de la ley de Moore para traer dispositivos inteligentes, conectados a cada persona en la tierra. En la misión se resalta cómo Intel sigue confiando y usando la Ley de Moore en la actualidad, esta ley establece que la cantidad de transistores en un procesador se duplicará cada 18 meses. Por otro lado, la ambición de la compañía se resalta aún más con la inclusión de "conectados a cada persona en la tierra", en donde deja en claro que su estrategia sigue apuntando a un mercado global en el segmento de semiconductores, microprocesadores y tecnologías relacionadas a la computación.

Visión: si es inteligente y está conectado, es mejor con Intel. En la visión se puede ver cómo Intel define los tipos de productos que pretende ofrecer a su mercado objetivo, seguido por un auto reconocimiento como la mejor compañía. En otras palabras, Intel se ve como la compañía mejor calificada y capaz de proporcionar productos inteligentes y conectados. Según Intel (2019), la ley Moore habla de que el número de transistores por pulgada en los circuitos integrados se duplicaría cada 18 meses y que esa tendencia continuaría al menos durante dos décadas. Aunque muchos piensan que la ley de Moore ya no es vigente, la misión de Intel nos deja en claro que la compañía sigue en búsqueda de la miniaturización de sus procesadores.

Intel fue el creador del primer microprocesador modelo 4004 del mundo en el año de 1969, llamándolo como la computadora en un chip. Para el año de 1980 se creó la arquitectura 8080, la cual fue elegida como la unidad de procesamiento central de la primera computadora personal de IBM (Encyclopedia Britannica, 2019). 
A mediados de la década de 1990, Intel se expandió más allá del negocio de los chips. Grandes fabricantes de computadoras personales (PC), como IBM y Hewlett-Packard, pudieron diseñar y fabricar computadoras basadas en Intel para sus mercados; sin embargo, Intel en su afán de incrementar su mercado buscó la forma de proporcionar su tecnología a compañías más pequeñas, lo cual ayudaría a expandir su mercado con mayor velocidad; para lograr esto, creó un sistema que contenía todas las partes necesarias de una computadora llamado tarjeta madre. Para 1995, Intel estaba vendiendo más de 10 millones de tarjetas madres a fabricantes de PC (Intel American Company, 2019).

A finales del siglo, Intel y chips compatibles de compañías como AMD se encontraban en todas las PC, excepto en Macintosh de Apple Inc., que había usado CPU de Motorola desde 1984. En 2005, Craig Barret, CEO de Intel en ese año, logró cerrar un contrato con Apple Inc., siendo el mismo Steve Jobs, director ejecutivo de Apple, quien diera el anunció: las futuras computadoras de Apple utilizarían las CPU de Intel, cubriendo así prácticamente todo el segmento de PC a nivel mundial (Intel American Company, 2019).

Intel domina el mercado de los procesadores y microprocesadores, pero al momento de hablar de dicha empresa se puede pensar en la gran variedad de segmentos a los que se enfoca y al gran número de competidores; sin embargo, este trabajo de investigación se estará enfocado principalmente en el segmento de los PC (laptops), en el cual Intel solo cuenta con un único competidor: Advanced Micro Devices (AMD).

AMD es una compañía de semiconductores con sede en Sunnyvale, California, que se dedica al desarrollo de procesadores, es el segundo fabricante a nivel mundial de procesadores $\mathrm{x} 86 \mathrm{y}$ el segundo fabricante de tarjetas gráficas para el sector profesional y el doméstico (AMD, 2019). Fue fundada el 1 de mayo de 1969 por un grupo de ejecutivos de Fairchild Semiconductor, entre ellos se encontraba Jerry Sanders III, Edwin Turney, John Carey, Steven Simonsen, Jack Gifford, Frank Botte, Jim Giles y Larry Sterfer (AMD, 2019). Actualmente AMD e Intel son las dos únicas compañías que venden procesadores x86.

\section{Conceptos}

\section{Estrategia}

Según Vargas, Guerra, Bojórquez y Bojórquez (2017), la estrategia se puede definir como plan, como acción o como integración o teoría. Esto significa que la estrategia es definida como el alineamiento o dirección que se da a los 


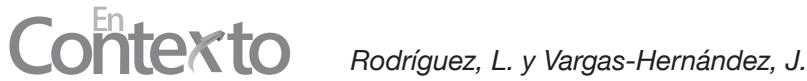

recursos internos de una organización para modificar, liderar, adaptarse y en el peor de los casos sobrevivir a las condiciones del medio ambiente. Esta definición permite que las empresas planeen mejor en el caso de que hubiera cambios, dando lugar a poder adaptarse o ajustarse a estos.

\section{Industria}

De acuerdo con Peng (2012), una industria es un grupo de firmas o empresas que produce bienes y/o servicios similares entre sí. Aunque también podría ser definida como un conjunto de empresas que oferta productos sustitutos cercanos (Hill \& Jones, 2004).

\section{Mercado}

La definición de mercado de Smith (2011), quien fue llamado el padre de la economía moderna, se basaba en que el egoísmo es la característica propia de los agentes económicos racionales que los motiva a realizar tratos, trueques y compras para obtener lo que necesitan de otros, a ese lugar físico lo llamó mercado. Sin embargo, con el efecto de la globalización y la innovación, las forma en que se pueden realizar las transacciones han cambiado y no solamente se realizan en un lugar físico, sino también por medio plataformas digitales haciendo conexión desde todas partes del mundo, esto solo se puede dar si se establecen precios entre compradores y vendedores (Banco de México, s.f.).

De acuerdo con Parkin y Loría (2010), el mercado es cualquier acuerdo que permite a compradores y vendedores obtener información sobre algún bien o servicio y hacer negocios entre sí. Aunque también puede ser definido como lo hace Vargas et al. (2017): el mercado es el mejor mecanismo para emparejar oferta y demanda, fijar precios y extraer la máxima utilidad de los recursos finitos.

\section{Oligopolio}

Según Varian (2010), un oligopolio es un mercado en el que hay algunas empresas que se dan cuenta de su interdependencia estratégica, puede comportarse de varias formas dependiendo del tipo exacto de interrelación. Las formas diferentes como operan un grupo de empresas que controlan la producción y el precio en un mercado pueden ser el oligopolio bilateral en el que solo hay pocos oferentes y pocos demandantes; el oligopsonio u oligopolio de demanda en el que hay muchos oferentes y pocos demandantes o consumidores: el Duopolio donde solo son dos empresas las que controlan el mercado. Los oligopolios también pueden ser concentrados, diferenciados o competitivos en la producción en un mercado. 


\section{Revisión teórica}

Conforme a la visión basada en la industria, Peng (2012) menciona que dicho enfoque estudia cómo las empresas se valen de las oportunidades y confrontan las amenazas de la industria, dicho de otra forma, es la manera de concursar o competir, siendo esta actividad la base de su diferenciación (Vargas et al., 2017).

La rivalidad generada por la competencia utiliza la combinación de cinco fuerzas que define la estructura de una industria y moldea la naturaleza de la interacción competitiva dentro de ella (Porter, 2008). Michel Porter quien propagó el modelo llamado las cinco fuerzas, las cuales forman la parte principal de la visión estratégica basada en la industria (Vargas et al., 2017).

Las cinco fuerzas de Porter son (como se citó en Vargas et al., 2017):

1. Rivalidad entre competidores: relacionado con el número de competidores, estos tienen que ser similares en su tamaño, en los productos que ofertan, la capacidad de cada industria, el lento crecimiento de la industria y en los altos costos de salida.

2. Amenaza de una entrada potencial: barreras a la entrada, las cuales aumentan los costos (economías de escala, know how) y lealtad de los clientes.

3. El Poder de negociación de los proveedores: capacidad para elevar los precios o calidad de los bienes y servicios, brindando productos únicos y pocos proveedores.

4. El Poder de negociación de los compradores: pocos compradores tienen fuerza en negociación, compran productos sin marcas específicas y que no producen un ahorro.

5. Amenaza de los productos sustitutos: son amenazantes siempre y aún más cuando son superiores en su calidad.

Porter (1981) plantea un marco para analizar el nivel de competencia dentro de una industria para que pueda desarrollarse en su entorno. Dicho esto, una estrategia eficiente debería cumplir los siguientes elementos clave: fortalezas y debilidades de la compañía, oportunidades económicas y técnicas y amenazas de la industria, valores personales de implementadores clave y expectativas sociales más amplias Porter (1981). 


\section{Análisis de resultados}

\section{Rivalidad entre competidores}

Según Semmler (2010), en cuanto a la industria del microprocesador, se sabe que Intel y AMD son los dos líderes del mercado mundial en la producción de microprocesadores. Intel tiene una cuota de mercado del 81,7\%, mientras que AMD tiene una cuota de mercado del 16,9\%. Intel es históricamente la más grande y la más antigua de las dos empresas.

Estas dos empresas compiten en las capacidades con respecto a los procesadores, tanto en el área de seguridad como en la velocidad de procesamiento. Dicha competencia se basa en el tamaño del procesador, la medida que se tiene de un procesador es de 14 nanómetros; por lo que las dos empresas saltaron a una nueva tecnología: fabricar un procesador más pequeño para alcanzar los 10 y 7 nanómetros para ofrecer mejor ventaja competitiva en el mercado. En la actualidad, las dos se encuentran en una competencia por hacer más pequeño dicho procesador y ofrecerlo con mayor rapidez en el mercado (Hruska, 2019).

A pesar de las rivalidades entre estas dos empresas, Intel sigue teniendo el liderazgo con respecto a AMD. Según Peña (2019), a pesar de que ambas compañías están produciendo procesadores que están a una distancia sorprendente en casi todos los frentes (precio, potencia y rendimiento, chips), Intel tienden a ofrecer un mejor rendimiento por núcleo. Según Semmler (2010), Intel puede definirse como un Oligopolio, con una empresa líder, y AMD como una seguidora o magistral. Respecto al tipo de competencia que tiene Intel con AMD, y a su estructura de mercado, no existe otro gran productor de microprocesadores y alrededor del $98.6 \%$ de la cuota del mercado total está en poder de las dos empresas.

Según Semmler (2010), las características típicas de este mercado oligopólico son:

1. Las empresas venden productos estandarizados.

2. Las empresas son establecedores de precios, lo que significa que pueden influir en la cuota de mercado del producto, estableciendo sus precios (Bertrand Oligopoly) o las cantidades y dejando que los precios reaccionen (el oligopolio de Cournot).

3. Hay grandes barreras de entrada. Las barreras de entrada a una industria o mercado son factores que lo hacen diculto o costoso para otras empresas 
recién llegadas. Tales barreras pueden ser legales, burocráticas, financieras o económicas.

4. Hay pocas empresas y hay interacciones estratégicas entre ellas.

5. La publicidad pesada y la denominación de marca, así como el uso de la tecnología se producen en el mercado con el fin de diferenciar los productos.

Solo existen dos empresas, y las barreras de entrada son los suficientemente grandes como para impedir que nuevas empresas entren en el mercado, estas barreras son las patentes que tiene cada una a lo largo de los años, además de los contratos con los fabricantes de PC y portátiles (Semmler, 2010).

Dicho esto, se puede mencionar que, a nivel mundial, Intel se puede ubicar en una estructura de mercado oligopólica, en especial como un duopolio, ya que, como se mencionó anteriormente, AMD es la única empresa en su rama que puede hacerle competencia sobre los procesadores que actualmente están en el mercado; es decir, existen solamente dos empresas, y uno de los supuestos que presenta una estructura Oligopólica es precisamente que existan pocas empresas en su mismo mercado. Sin embargo, en este caso, el Oligopolio también tiene un cuarto supuesto, en donde se puede decir que las empresas tienen interacción estratégica entre ellas, pero este no es el caso, porque todas las herramientas con las que cuenta tanto Intel como AMD son estrictamente confidenciales.

El compartir información de sus procesos e interactuar de una manera estratégica entre ellas, podría convertirse en una práctica monopólica llamada colusión e Intel (2019), por cuestiones éticas, no la realiza (Semmler, 2010). En 1976 AMD e Intel firmaron un acuerdo de licencia cruzada que eventualmente conduce a la eliminación de otros competidores debido a una brecha tecnológica creciente, pero en 1987 se termina el acuerdo de licencias cruzadas entre AMD e Intel. Esto marca el comienzo de una fuerte competencia entre las dos empresas (Semmler, 2010).

\section{Resultados financieros}

En el aspecto económico, podemos analizar las ganancias que ambas compañías tuvieron en 2018, según los reportes financieros de Intel, en 2018 la compañía obtuvo 70.8 mil millones de dólares siendo esto un incremento del 13\% con respecto a lo reportado en 2017 (Intel, 2019); por su lado, AMD (2019) reportó un ingreso anual en 2018 de 6.48 mil millones de dólares (23\% más que en 2017). 


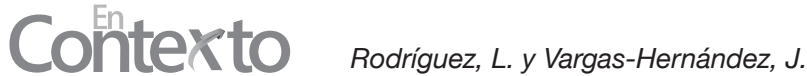

\section{Amenaza de una entrada potencial}

Las economías surgen en el momento en que las empresas producen volumen y gozan de precios bajos por unidad, además de que tienen mayor tecnología y eficiencia para poder ser competitivas asegurándose de que ningún competidor pueda entrar al mercado (Porter, 2008).

Según estudios realizados por el Instituto Tecnológico de Massachusetts (Mims, 2010), el costo para la construcción de una planta de fabricación de microprocesadores es de alrededor de 3 y 5 mil millones de dólares, lo cual es un costo elevado que obliga a las empresas a mantener en funcionamiento al ciento por ciento de sus fábricas para poder recuperar la inversión. Según el Mims (2010), en la industria de los microprocesadores sólo Samsung e Intel cuentan con un volumen de producción elevado que les permite costear dichas plantas de producción de última generación, por lo que otras compañías de este medio como AMD se ven en la necesidad de contratar fábricas externas, como Global Foundries y Taiwan Semiconductor Manufacturing Company, para la producción de sus procesadores.

La necesidad de tener que invertir grandes cantidades de dinero en instalaciones con el fin de poder competir, implica asumir las pérdidas iniciales de una empresa cuando empieza (Porter, 2008).

En los análisis del Institute Pamore (Ferguson, 2017) se hace referencia a las limitantes que la industria tiene: el acceso a dichas fábricas, el alto costo de construcción y mantenimiento; eso impide que empresas más pequeñas como ARM Holding manufacturera de procesadores, puedan aspirar a tener una planta para la fabricación de sus propios dispositivos, siendo esta una de las principales barreras que otras compañías tienen que enfrentar para poder ingresar al amplio mundo de las computadoras personales y servidores.

Una de las estrategias basadas en la ley de Moore es disminuir el tamaño del procesador, este cambio conlleva una gran inversión, ya que se tiene que construir una nueva fábrica que cuente con las capacidades y herramientas necesarias para lograr este nuevo tamaño, lo que implica una ventaja competitiva de Intel con respecto a su competencia, ya que el tiempo de fabricación y costo de sus competidores es aún mayor (Berkeley Economic Review, 2019). 


\section{Poder de negociación de los proveedores}

Una empresa de gran tamaño, como Intel, es difícil que cambie de proveedor, ya que se gastan grandes sumas de dinero en equipamiento especializado y en la fabricación del producto (Porter, 2008).

No existe sustituto alguno del producto que se ofrece, ya que por su alta innovación es difícil que un comprador pueda rechazar el producto y, por ende, los proveedores tendrán más influencia en la negociación, ya que ninguna otra industria lo podría tener (Porter, 2008).

\section{Poder de negociación de los compradores}

Los compradores pueden llegar a ser poderosos si cuentan con influencia negociadora sobre la industria, estos pueden ser influyentes negociador si existen pocos compradores y si su compra es en gran volumen; otra influencia es que los compradores se enfrentan a pocas variaciones de los costos cuando cambian de vendedor; además de amenazar con retirarse y producir ese mismo producto si otros vendedores resultan ser más rentables (Porter, 2008).

Sin embargo, en el caso de Intel existen factores que alteran las cinco fuerzas de Porter en el aspecto de la negociación con los clientes, dichos factores benefician a la marca Intel, puesto que al haber baja disponibilidad de sustitutos (en el caso de computadoras personales solo AMD es competencia), los compradores se ven en la necesidad de continuar consumiendo productos de Intel, aunado a los altos costos de conmutación que existen entre los actuales productos alternos. Intel (2019) refiere que durante muchos años su alianza con Microsoft ha ayudado a que el primero pueda tener un dominio en el mercado de computadoras y, sobre todo, en los nuevos segmentos que ya empiezan a crecer de manera exponencial como lo es la nube, $5 \mathrm{G}$ e inteligencia artificial. Wired (2017) menciona que esta alianza, que data de principio de los 80 , influye en la negociación de los clientes con Intel, ya que si Microsoft quisiera sustituir los actuales procesadores tendrían muchos problemas de compatibilidad con productos anteriores.

Compañías como Microsoft y Apple carecen de instalaciones de fabricación de microprocesadores, así como empresas que pueden proveer dichos sustitutos carecen de igual forma de fábricas, lo que limita su capacidad de producción en volúmenes altos, es por esto que los clientes ejercen una fuerza débil en el entorno de la industria de Intel, permitiéndole tener un poder alto en las negociaciones. 


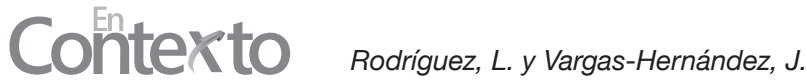

Intel cuenta con fábricas para producción de microprocesadores en todo el mundo, si nos referimos a Estados Unidos, cuenta con 4 fábricas ubicadas en (Intel, 2019):

a) Chandler, Arizona.

b) Hudson.

c) Rio Rancho.

d) Hillsboro, Oregón.

Fuera de Estados Unidos:

e) Leixlip, Irlanda.

f) Jerusalem, Israel.

g) Kiryal, España.

h) Dalian, China.

Los sitios de producción fuera de los Estados Unidos se encuentran en localidades que le permiten tener una producción y distribución cercana a los distintos centros de investigación y desarrollo, así como a sus clientes alrededor del mundo. Los centros de investigación y desarrollo se pueden encontrar tanto dentro como fuera de Estados Unidos (Intel, 2019).

Oficinas de Intel dentro de Estados Unidos:

a) Oregón

b) Santa Clara

c) Austin

d) Chandler

e) Folsom

Oficinas de Intel fuera de los Estados Unidos:

$\begin{array}{llll}\text { Argentina } & \text { Dinamarca } & \text { Italia } & \text { Rumania } \\ \text { Australia } & \text { Egipto } & \text { Japón } & \text { Federación Rusa } \\ \text { Austria } & \text { Finlandia } & \text { Letonia } & \text { Singapur } \\ \text { Bélgica } & \text { Francia } & \text { Malasia } & \text { Corea del Sur } \\ \text { Canadá } & \text { Alemania } & \text { México } & \text { Suecia } \\ \text { Chile } & \text { India } & \text { Países Bajos } & \text { Suiza } \\ \text { China } & \text { Indonesia } & \text { Perú } & \text { Taiwán } \\ \text { Costa Rica } & \text { Irlanda } & \text { Polonia } & \text { Reino Unido } \\ & \text { Israel } & \text { Portugal } & \text { Vietnam }\end{array}$




\section{Amenaza de productos sustitutos}

Con la aparición de nuevas tecnologías y la innovación constante, estas pueden actuar como productos sustitutos de los microprocesadores. En consecuencia, a estos cambios constantes Intel, a diferencia de AMD, ha tratado de implementar una estrategia que le permita garantizar una ventaja competitiva y reinventarse en torno a la innovación y tendencia que actualmente avanzan a pasos agigantados, adentrándose al mundo de la tecnología 5G, la inteligencia artificial, la computación cuántica y desarrollos con mayor tecnología, como lo es un coche autónomo. Sin embargo, actualmente el desarrollo de procesadores sigue siendo uno de los mayores negocios con los que cuenta Intel, con ello esta compañía se posiciona como un líder dentro del mercado en competencia única con AMD, ya que sigue enfocándose en el desarrollo de los microprocesadores (Jiménez, 2018).

Los cambios tecnológicos tan acelerados que se tienen hoy, como la amenaza de sustitutos, la falta de innovación o pensar en implementa nuevas estrategias, haría que Intel se vería resentida, y esto podría llevar a que el rendimiento de una industria se vea limitada en su potencial (Porter, 2008).

\section{Conclusiones}

Intel es una empresa que, desde sus inicios, en 1968, se ha ido posicionando en el mercado de los microprocesadores, confiando en la ley Moore; esto le ha servido para seguir afianzándose en el mercado mundial. Intel Corporation cuenta con un competidor a nivel mundial, el cual es Advanced Micro Devices (AMD), una compañía que fue fundada un años después de Intel, y representa la única rival dentro del mercado de microprocesadores, ya que es considerada como la segunda fabricante a nivel mundial. Ambas empresas compiten en las capacidades de sus procesadores tanto en el área de seguridad como en lo que atañe a la velocidad de procesamiento.

Dicha investigación se enfoca, primordialmente, en una visión basada en la industria, pero deja muy en claro que no podemos pensar en ella sin pensar en las cinco fuerzas de Michael Porter, o también llamadas los diamantes de Porter, las cuales nos indican que una empresa puede tener ventajas competitivas, como:

Rivalidad entre competidores: la industria deja en claro que Intel es más sobresaliente que AMD, ya que la primera abarca un mercado de $81.7 \%$ y la segunda uno de 16.9\% (Semmler, 2010; Intel, 2019 y AMD, 219), lo que hace 


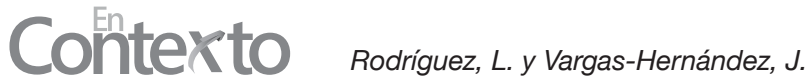

que Intel se coloque como el número uno; de igual forma, en la parte financiera nos podemos dar cuenta que Intel sigue estando como líder, porque sus reportes, para el 2018, son prácticamente diez veces mayores que los de AMD.

Amenaza de una entrada potencial: Intel ha invertido en plantas de fabricación en varias partes del mundo, lo que hace que disminuyan sus costos de producción; es por ello que las empresas que pretendan entrar a la competencia del mismo mercado serán sacadas, ya que el costo es demasiado elevado y requerirán sostenerse si hubiera perdidas; de allí que Intel se cubra con una gran inversión.

El poder de negociación de los proveedores: cuando el proveedor tiene el control de dicha negociación, sea por tener la mejor calidad, seguridad, velocidad o por todas las características idóneas que el cliente busca, este no puede tener más opciones $\mathrm{y}$, por ende, consume directamente de la compañía.

El poder de negociación de los consumidores: los consumidores pueden llegar a tener mucha influencia en este aspecto, ya que, si la empresa proveedora tiene alguna competencia que pueda ofrecer algún producto sustituto o un producto con las mismas características y con precios más bajos, el consumidor puede amenazar con retirarse y buscar a alguien más, por lo que Intel siempre deberá estar un paso adelante de la innovación y sentido de servicio al cliente, para poder seguir teniendo a los consumidores satisfechos.

Amenaza de los productos sustitutos: la innovación es una herramienta primordial para la empresa, ya que esta puede ser su amenaza principal; es por ello que Intel, dando un paso más hacia la innovación, y diferenciándose de la competencia, decidió lazarse también al mundo de la tecnología 5g, inteligencia artificial y computación cuántica, lo que le permitiría, en un futuro, poder ser una de las empresas con mayor tecnología y, sobre todo, ser más rentable para toda la industria tecnológica.

Con el análisis previo realizado a Intel Corporation, podemos concluir que Intel es la compañía líder en el segmento de semiconductores, debido a sus ganancias financieras y el mercado que actualmente abarca. AMD es la única compañía en realizar un esfuerzo por alcanzar un mayor mercado en el segmento de computadoras personales, así mismo empresas como ARM han intentado ingresar sin éxito.

Realizando un análisis con las cinco fuerzas de Porter, y especificando el factor de los productos sustitutos o la amenaza de entrada potencial, hemos hallado 
que existen distintas barreras para que una compañía pueda competir en este gran segmento, las barreras más importantes son:

1. Alianzas con fabricantes de PC.

2. Alto costo de creación y mantenimiento de las fábricas de producción.

3. Incompatibilidad con sistemas anteriores.

4. Alto costo de conmutación de un nuevo procesador por parte de los fabricantes de PC.

Una empresa pequeña que no cuente con el capital financiero para invertir en fábricas de producción de procesadores ve disminuida sus posibilidades de éxito, ya que no será capaz de solventar la demanda actual de parte de los compradores como Apple Inc., Microsoft, Dell, etc., esto también complica que los compradores puedan realizar alianzas estratégicas con empresas más pequeñas.

Intel tiene a través del mundo distintas oficinas y fábricas que dan mayor seguridad a los compradores en cuanto a su capacidad de producción, así como de soporte. Intel cuenta con más de cincuenta localidades a nivel mundial, lo cual le permite brindar soporte en diferentes regiones. La capacidad de negociación con las compañías líderes en producción de computadoras personales, laptops y de escritorio ha permitido a Intel mantener el volumen de venta desde mediados de 1980, estas alianzas incluyen a Microsoft y Apple, las cuales tienen un dominio de mercado elevado. La compatibilidad de los procesadores de Intel con las versiones anteriores de equipos de cómputo permite ofrecer a Intel una transición transparente hacia las nuevas tecnologías.

Las empresas que deseen ingresar a este mercado se enfrentarán a distintos retos para poder disminuir el mercado que Intel actualmente domina, sumado a que en la actualidad Intel ya se encuentra incursionando en segmentos nuevos con la clara misión de dominarlos proveyendo tecnología de punta como lo es el 5G, la inteligencia artificial y la nube.

\section{Referencias}

AMD. (2019). About AMD. Recuperado de https://www.amd.com/en/corporate/ about-amd

Banco de México. (s.f.). Glosario. Recuperado de http://www.anterior.banxico. org.mx/divulgacion/glosario/glosario.html\#M 


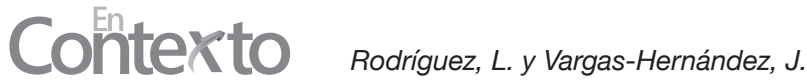

Berkeley Economic Review. (2019). Intel and AMD market competition. Recuperado de https://econreview.berkeley.edu/intel-and-amd-marketcompetition/

Ferguson, E. (2017). Intel Corporation's SWOT Analysis \& Recommendations. Recuperado de http://panmore.com/intel-corporation-swot-analysisrecommendations

Hill, C. \& Jones, G. (2004). Strategic management theory an integrated approach. Texas, United States: Houghton Mifflin.

Hruska, J. (2019). Intel will Fab ARM chips for LG on upcoming $10 \mathrm{~nm}$ foundry node. Recuperado de https://www.extremetech.com/computing/233886intel-will-fab-arm-chips-for-lg-on-upcoming-10nm-foundry-node

Intel American Company. (2019). Encyclopedia Britannica. Recuperado de https://www.britannica.com/topic/Intel

Intel. (2019). ¿Cuál es la declaración de misión, los valores y los objetivos de Intel? Recuperado de https://www.intel.la/content/www/xl/es/support/ articles/000015119/programs.html

Jiménez, A. (2018). Intel: 50 años de la empresa que cambio al mundo. El mundo. Recuperado de https://www.elmundo.es/papel/historias/2018/07/18/5b4dccd422601d313e8b46e1.html

Mims, C. (2010). Retos de la industria. El alto coste de cumplir la Ley Moore. Recuperado de https://www.technologyreview.es/s/1128/retos-de-laindustria-el-alto-coste-de-cumplir-la-ley-de-moore

Parkin, M. y Loría, E. (2010). Microeconomía versión para Latinoamérica. México: Pearson.

Peng, M. (2012). Global Strategy. Cincinnati, United States: Thomson SouthWestern.

Peña, M. (2019). Procesador AMD \& Intel: los enfrentamos en esta guía. Recuperado de https://es.digitaltrends.com/computadoras/amd-vs-intel/

Porter, M. (1981). The Contributions of Industrial Organization to Strategic Management. The Academy of Management Review, 6(4), 609-620. Recuperado de http://www.jstor.org/stable/257639 
Porter, M. (2008). Ser competitivo. España: Ediciones Deusto. Recuperado de https://www.planetadelibros.com/libros_contenido_extra/35/34984_ Ser_competitivo.pdf

Semmler, A. (2010). Competition in the microprocesador market Intel, AMD and Beyond. University of Trier, Germany. Recuperado de https://www. academia.edu/1860422/Competition_in_the_Microprocessor_Market_ Intel_AMD_and_Beyond

Smith, A. (2011). La riqueza de las naciones. México: Ediciones Brontes.

Vargas, J., Guerra, E., Bojórquez, A. y Bojórquez, F. (2017). Análisis de la gestión estratégica. España: Heriberto Daniel Camacho Fernández.

Varian, H. (2010). Microeconomía intermedia. Un enfoque actual. Barcelona, España: Editorial Antoni Bosch.

Wired. (2017). Microsoft and Intel's decades-long Alliance has started to fray. Recuperado de https://www.wired.com/2017/03/wintel-going-notdead-yet/

\section{Para citar este artículo:}

Rodríguez, L. y Vargas-Hernández, J. (2020). Ventaja competitiva de INTEL en el segmento de los microprocesadores. En-Contexto, $8(12), 135-151$. 